\title{
Iterative Learning-Based Admittance Control for Autonomous Excavation
}

\author{
Heshan Fernando • Joshua A. Marshall . \\ Johan Larsson
}

Received: June 14, 2019

\begin{abstract}
This paper presents the development and field validation of an iterative learning-based admittance control algorithm for autonomous excavation in fragmented rock using robotic wheel loaders. An admittance control strategy is augmented with iterative learning, which automatically updates control parameters based on the error between a target bucket fill weight and the measured fill weight at the end of each excavation pass. The algorithm was validated through full-scale autonomous excavation experiments with a 14-tonne capacity load-hauldump (LHD) machine and two different types of excavation materials: fragmented rock and gravel. In both excavation scenarios, the iterative learning algorithm is able to update the admittance control parameters for a specified target bucket fill weight, eliminating the need to manually re-tune control parameters as material
\end{abstract}

This work was supported in part by the Natural Sciences and Engineering Research Council of Canada (NSERC) under project RGPIN-2015-04025, the Swedish Knowledge Foundation (KK-stiftelsen) under project 20150282, and by Epiroc Rock Drills AB (Sweden).

This work was completed while the second author was a KKS International Visiting Professor at the Centre for Applied Autonomous Sensor Systems (AASS) in the School of Science and Technology at Örebro University, Sweden.

H. Fernando

Department of Mechanical and Materials Engineering

Queen's University at Kingston, Canada

Tel.: +1-613-533-2575

E-mail: h.fernando@queensu.ca

J. A. Marshall

Faculty of Engineering and Applied Science

Queen's University at Kingston, Canada

Tel.: +1-613-533-2921

E-mail: joshua.marshall@queensu.ca

J. Larsson

Rocktec Division

Epiroc Rock Drills AB at Örebro, Sweden

Tel.: +46-19-6707262

E-mail: johan.larsson@epiroc.com 
characteristics change. These results have practical significance for increasing the autonomy of robotic wheel loaders used in mining and construction.

Keywords Autonomous excavation · iterative learning · admittance control · mining robotics.

\section{Introduction}

An autonomous excavation system where a machine, such as a robotic wheel loader, performs an excavation task itself using sensory feedback has obvious applications in mining, construction and space exploration/development, where humans cannot be present for safety, logistical, and/or cost reasons. Despite many years of research into automated earth-moving [3], a commercially viable autonomous excavation system has not yet seen widespread use. The challenge is that excavation performance is strongly influenced by the conditions of interaction between the machine and the environment, which are unknown and changing throughout the excavation process. Thus, a practical control strategy for autonomous excavation requires learning and adaption in order to achieve consistent bucket filling performance in variable conditions.

Recent research $[7,8]$ has shown that an admittance-based control strategy for autonomous excavation, which utilizes force feedback to regulate velocity commands to a loader's bucket actuator, achieves consistent bucket filling performance; however, certain control parameters require tuning when the excavated media changes characteristics (e.g., pile fragmentation, water content, etc.). In practice, material characteristics are unknown and changing throughout an excavation operation. Thus, the admittance controller requires extension through learning and adaptation for successful deployment in real excavation scenarios.

In this study, an admittance controller is augmented with iterative learning to update control parameters after each excavation pass, based on the error between a target bucket fill weight and the measured fill weight from the previous pass. The proposed algorithm was validated through full-scale excavation experiments by using a 14-tonne capacity load-haul-dump (LHD) machine, shown in Figure 1, at an underground mining test facility located near Kvarntorp, Sweden.

\subsection{Related Work}

Autonomous excavation has been researched for many decades $[11,3]$, but a commercially viable system for autonomous excavation has not yet seen widespread use in mining or construction. Motion control strategies that regulate bucket motion to follow a pre-determined path are ineffective when excavating heterogenous materials, such as fragmented rock [15], because subsurface obstacles in heterogeneous material can cause large position errors that result in saturated actuation. AI-based approaches [4] that use artificial neural networks (ANNs), trained with imperfect expert operator data to determine actuator inputs during autonomous operation, are also not practical because these approaches require significant amounts of data to train and can exhibit unpredictable behaviour or poor performance in untrained situations. 


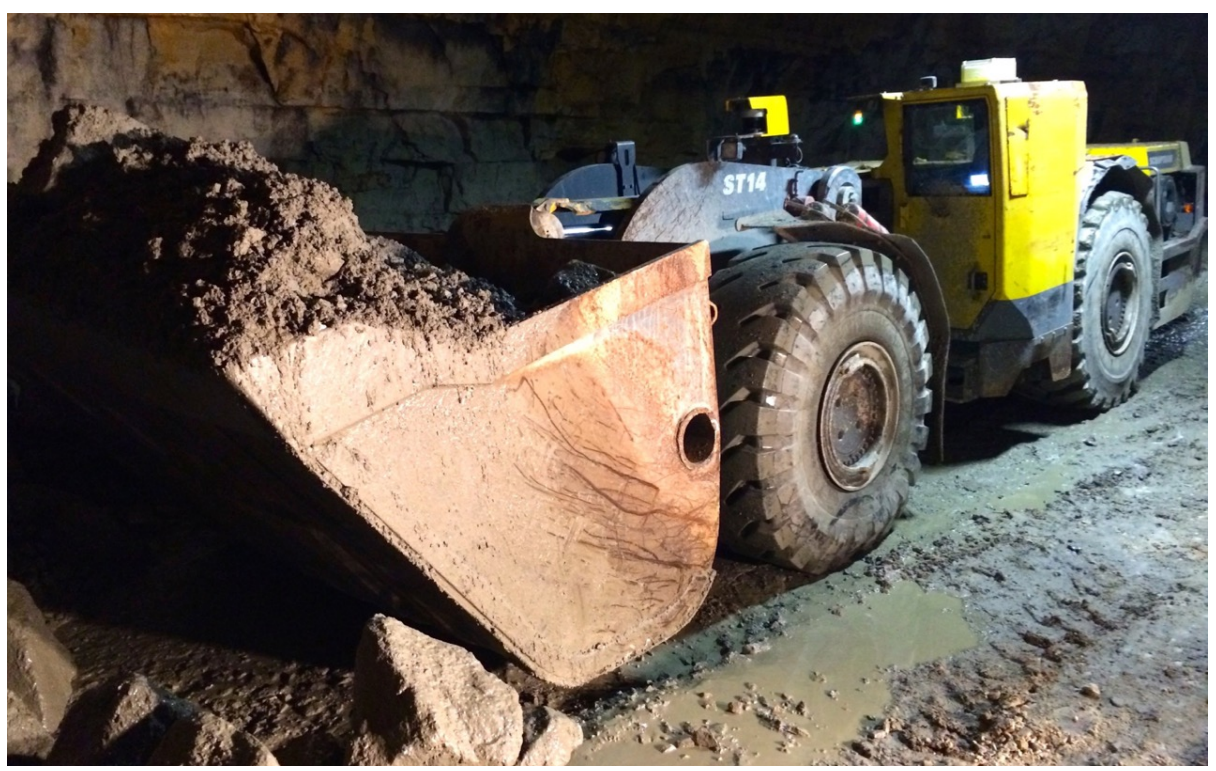

Fig. 1 An Epiroc ST14 load-haul-dump (LHD) machine, which is a type of wheel loader used for excavating fragmented rock in underground mining.

One promising approach to autonomous excavation is an admittance-based interaction control strategy that has been developed through extensive field experiments $[16,7,8]$. In contrast to pure motion control, which rejects disturbance forces to track a given motion reference trajectory, admittance control attempts to comply robot motion with environment interaction and react quickly to measured interaction forces by rapidly modifying the robot's reference velocity [17]. This approach has become popular in recent robot interaction applications such as robotic surgery [13], human-robot cooperation [10], and rehabilitation and therapy robotics [12]. Tuning admittance controllers is challenging because performance depends on the coupled interaction between a robot and a changing environment, which is difficult to model and identify correctly [18]. Thus, current research focuses on extending admittance controllers toward learning and adaptive controllers to ensure stable performance of robot interaction tasks with unknown and changing environments $[20,19,6,14,2]$.

\subsection{About this Paper}

Section 2 describes the admittance-based control strategy for autonomous excavation and provides an overview of iterative learning control and its application to autonomous excavation. Section 3 describes the experimental setup. Section 4 presents field results in a representative environment. 


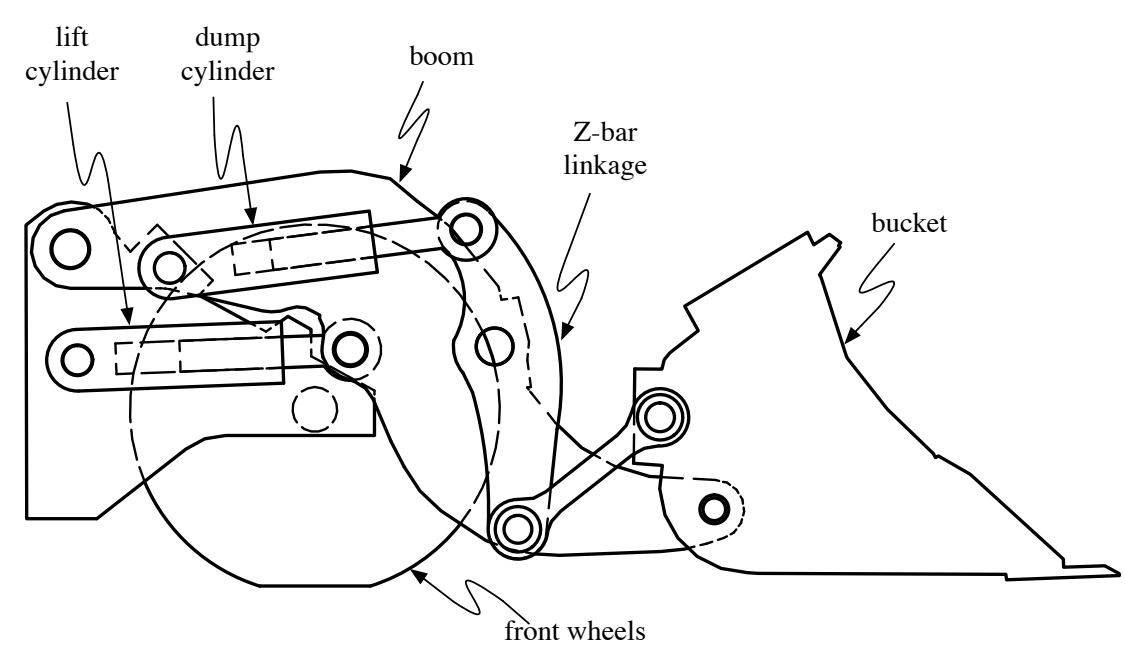

Fig. 2 Geometry of an Epiroc ST14 LHD machine's loading mechanism.

\section{Controller Development}

In this study, an admittance control strategy for autonomous excavation $[16,7$, $8]$ is augmented with iterative learning to enhance performance when excavating material with unknown or changing characteristics. This section provides an overview of admittance control and of Iterative Learning Control (ILC), which are used to formulate an iterative learning-based admittance control algorithm for autonomous excavation. This algorithm was validated through full-scale field experiments, which are described in Section 4.

\subsection{Admittance Control for Autonomous Excavation}

The focus of the current study is on autonomous excavation using load-haul-dump (LHD) machines, which are used for excavating fragmented rock in underground mining. The loading mechanism of a typical LHD machine consist of a hydraulically actuated boom and bucket as shown in Fig. 2. The dump cylinder actuates the bucket through a Z-bar linkage, so the dump cylinder extension corresponds to the bucket loading motion, and the dump cylinder retraction corresponds to the bucket dumping motion. Two parallel lift cylinders actuate the boom, which lift or lower the bucket (e.g., when dumping material onto a truck bed). The objective of the excavation task is to effectively regulate inputs to these actuators to allow the bucket to dig through a pile and fill the bucket to a target weight at each excavation pass.

A block diagram of the admittance control strategy for autonomous excavation is shown in Fig. 3. The controller is implemented in the loader's actuator space, which consist of the throttle, dump cylinder and lift cylinders. Only the dump cylinder is controlled during digging. Tractive effort is supplied through a constant input to the throttle actuator $u_{t}=\bar{u}_{t}$. In [16], it was noted that "any tractive 


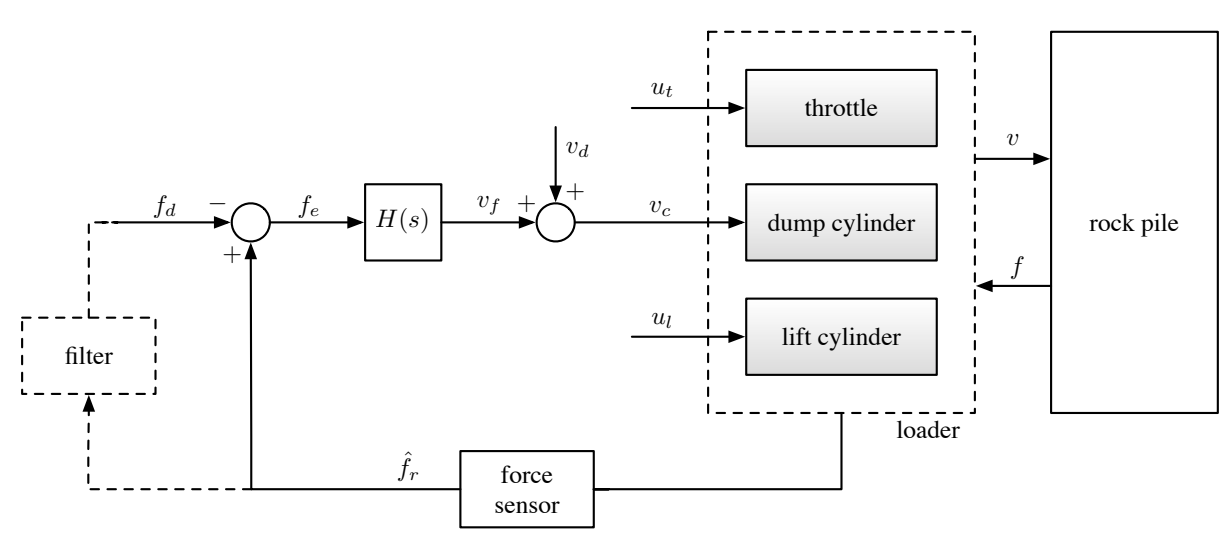

Fig. 3 A block diagram of the admittance-based control strategy for autonomous excavation using robotic wheel loaders.

effort supplied by the wheel loader is generally constant throughout the excavation operation, and that control of the tractive effort would not provide any significant contribution to the development of an autonomous excavation system". The lift cylinders are not actuated $u_{l}=0$; thus, hydraulic pressure measurements at the lift cylinders can be used as a force sensor to estimate the bucket-rock interaction forces $\hat{f}_{r}$, which serve as feedback to the dump cylinder admittance controller [7].

The dump cylinder admittance controller transfer function $H(s)$ can be expressed as a mechanical admittance $H(s)=v_{f}(s) / f_{e}(s)$, where the force error $f_{e}=\hat{f}_{r}-f_{d}$ is obtained by subtracting a nominal target force setpoint $f_{d}$ from the measured interaction force $\hat{f}_{r}$ [16]. The admittance controller $H(s)$ computes a velocity change $v_{f}$, which is combined with a reference velocity $v_{d}$ to produce a new velocity command $v_{c}=v_{d}+v_{f}$. It is assumed that a position-controlled dump cylinder is able to regulate the velocity as commanded.

Effective digging can be achieved by prescribing a constant admittance $H(s)=$ $K_{A}>0$, a constant reference velocity $v_{d}=\bar{v}_{d}$, and a target force $f_{d}$ obtained by filtering the measured force signal $\hat{f}_{r}$ [8]. In this implementation, the dump cylinder extends at a constant nominal rate $\bar{v}_{d}$, which increases by a rate $v_{f}$ when $\hat{f}_{r}>f_{d}$ (e.g., if a subsurface obstacle is encountered), resulting in faster bucket curl; and $\bar{v}_{d}$ decreases by a rate $v_{f}$ when $\hat{f}_{r}<f_{d}$ (e.g., if machine loses contact with the pile) resulting in slower bucket curl. This behaviour is dependent on proper selection of the target force $f_{d}$.

For ideal digging, the target force should follow the nominal level of interaction so the dump cylinder admittance controller can react to sudden increases and decreases in the forces appropriately. The nominal level of interaction forces is difficult to predetermine because it is affected by several factors, such as the characteristics of the media, amount of material in the bucket, orientation of the bucket, and throttle. In our earlier work [8], we showed that the target force can be obtained by filtering the measured forces $\hat{f}_{r}$ with a windowed median filter. Given a discrete-time implementation of the control system, at a time step $k$, the target force filter is defined as

$$
f_{d, k}=\operatorname{median}\left(\hat{f}_{r, k-1}, \hat{f}_{r, k-2}, \ldots, \hat{f}_{r, k-N}\right)
$$


where $N>0$ is the size of the window (an odd integer). Thus, the target force closely follows the measured forces with a lag, which is determined by $N$. The median filter permits sudden changes in the measured forces, without the measured forces significantly affecting the nominal force level.

In this control strategy, there are four parameters to tune: $K_{A}, N, \bar{u}_{t}$ and $\bar{v}_{d}$. In our earlier work [8], we determined that the values of the admittance gain $K_{A}$ and median filter window size $N$ only affect the level of response to changing forces. Higher $K_{A}$ values result in higher velocity commands to the dump cylinder. This parameter must be tuned to minimize actuator saturation. Higher $N$ results in a greater lag for the target force $f_{d}$, resulting in a positive-biased velocity change $v_{f}$. The window size $N$ must be tuned such that $f_{d}$ closely follows $\hat{f}_{r}$. In our experiments, we found that changing $K_{A}$ and $N$ do not significantly contribute to bucket fill. Tuning throttle input $\bar{u}_{t}$ and dump cylinder reference velocity $\bar{v}_{d}$ have the greatest impact on bucket fill weight. Thus, these two parameters must be learned and adapted for the excavation conditions.

\subsection{Iteratively Learning to Dig}

"Iterative Learning Control (ILC) is based on the notion that performance of a system that executes the same task multiple times can be improved by learning from previous executions (trials, iterations, passes)" [1]. For example, consider a robotic system that repeatedly performs a tracking task under the same operating conditions. We can model this system at pass $j$ as

$$
\mathbf{y}_{j}=\mathbf{G u}_{j}+\mathbf{d},
$$

where $\mathbf{y}_{j}$ is the robot's coordinates, $\mathbf{u}_{j}$ are its inputs and $\mathbf{d}$ are unmodeled disturbances (e.g., friction). The objective is to reduce the tracking error $\mathbf{e}_{j}=\mathbf{y}_{j}-\mathbf{y}_{d}$. A conventional controller yields the same tracking error at each pass. In contrast, ILC iteratively improves tracking performance by incorporating the error information $\mathbf{e}_{j}$ from one pass into the control input $\mathbf{u}_{j+1}$ for subsequent passes. The simplest ILC formulation is a proportional (or P-type) learning function

$$
\mathbf{u}_{j+1}=\mathbf{u}_{j}+\mathbf{K} \mathbf{e}_{j},
$$

where $\mathbf{K}$ is a learning gain parameter that must be suitably designed such that $e \rightarrow 0$ as $j \rightarrow \infty$. In this case, the iteration dynamics can be described as

$$
\mathbf{y}_{j+1}-\mathbf{y}_{j}=\mathbf{G}\left(\mathbf{u}_{j+1}-\mathbf{u}_{j}\right) .
$$

Substituting (3) into (4) and rearranging yields

$$
\mathbf{y}_{j+1}=\mathbf{y}_{j}+\mathbf{G}\left(\mathbf{K e}_{j}\right) .
$$

Next, subtracting the target trajectory $\mathbf{y}_{\mathbf{d}}$ from both sides of (5) yields the system's error dynamics

$$
\mathbf{e}_{j+1}=(1+\mathbf{G K}) \mathbf{e}_{j} .
$$

Thus, for asymptotic stability

$$
|1+\mathbf{G K}|<1
$$


If the system model $\mathbf{G}$ is known, $\mathbf{K}$ can be designed so that the errors are asymptotically stable. If the system model is not known, then $\mathbf{K}$ must be tuned through trial and error on the real system.

To apply ILC to autonomous excavation, we first define the task error $e_{j}$ and system inputs $\mathbf{u}_{j}$ at each excavation pass $j$. To do this, we consider the autonomous excavation task as a tracking problem, where the objective of the system is to track a target bucket fill weight $W_{d}$ at each excavation pass. Thus the task error is defined as the difference between the actual bucket fill weight $W_{j}$ and the target bucket fill weight $W_{d}$

$$
e_{j}=W_{j}-W_{d}
$$

The dump cylinder reference velocity input $\bar{v}_{d}$ and throttle input $\bar{u}_{t}$ contribute most to bucket fill weight. Therefore, we postulate two P-type learning functions, based on (3), that iteratively update the two input parameters based on the bucket fill weight error:

$$
\begin{aligned}
& \bar{v}_{d, j+1}=\bar{v}_{d, j}+\alpha\left(W_{j}-W_{d}\right) \\
& \bar{u}_{t, j+1}=\bar{u}_{t, j}+\beta\left(W_{j}-W_{d}\right)
\end{aligned}
$$

This formulation introduces two learning gains $\alpha$ and $\beta$, which must be suitably designed to achieve stable learning behaviour. Low learning gain values will result in slower convergence for $W_{j} \rightarrow W_{d}$, but high learning gain values can cause unstable learning behaviour.

\section{Experimental Setup}

The iterative learning-based admittance controller for autonomous excavation was validated through full-scale field experiments with a 14-tonne capacity load-hauldump (LHD) machine at an underground test facility located near Kvarntorp, Sweden. The experimental set-up is depicted in Fig. 4.

\subsection{LHD Machine}

The Epiroc ST14 LHD machine can carry 14-tonnes, has a $335 \mathrm{hp}$ engine and a load-sensing hydraulic system. Fluid flow into the lift and dump cylinders is controlled by closed-centre electro-hydraulic servo-valves. The ST14 LHD can be equipped with different buckets. Typically, the bucket size is specified to maximize the carrying capacity of the LHD machine based on the bulk density of the material to be excavated. However, only two buckets were available at the test facility: 5.4 $\mathrm{m}^{3}$ and $7.8 \mathrm{~m}^{3}$. Both were used for experiments. The ST14 LHD is also equipped with control, sensing and actuation devices necessary to implement the proposed control system. The relevant systems and hardware are described below.

1. Pressure Transducers: Pressure transducers at the head $(h)$ and rod $(r)$ sides of one of the lift cylinders were used to measure the hydraulic pressures. Pressure measurements $P$, along with the cylinder head and rod cross-sectional areas $A$, were used to estimate the interaction forces $\hat{f}_{r}=P_{h} A_{h}-P_{r} A_{r}$.

2. Position Transducer: A wire potentiometer within the dump cylinder was used to measure its extension length. 


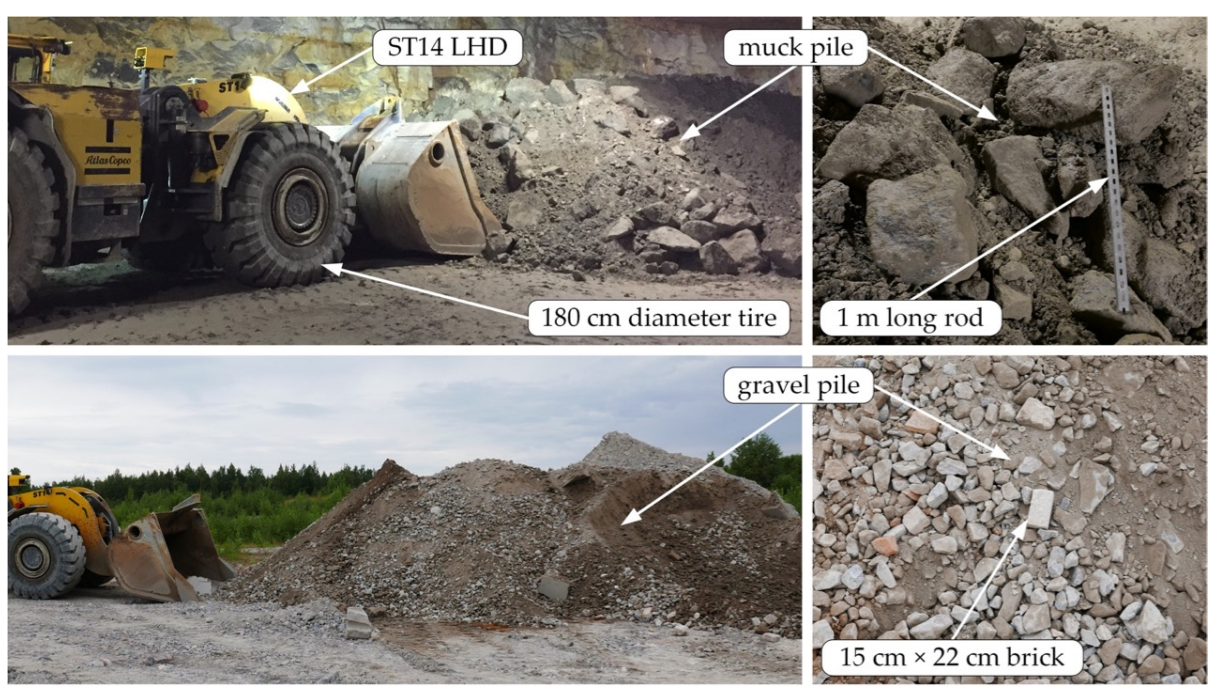

Fig. 4 Rock and gravel piles used for full-scale experiments. The Epiroc ST14 LHD is also shown to provide a sense of scale.

3. Control System: An on-board control system ran in real-time at a frequency of $20 \mathrm{~Hz}$ for reading sensors, sending actuator commands, and logging data.

4. Load Weighing System: A proprietary system calculated the bucket payload weight using a static load analysis as the LHD's boom was raised to a specific angle (measured with an absolute encoder at the boom joint) [9]. Lift cylinder pressure measurements were used to obtain the forces required for the analysis.

5. Electrohydraulic Servo Valves: Fluid flow to the lift and dump cylinders were controlled using servo valves. Valve dead-bands were reduced in the control software so that the valve commands corresponded to a near-linear response from the cylinder.

\subsection{Excavation Media}

A rock pile located underground and a gravel pile located at the surface of Epiroc's test facility near Kvarntorp, Sweden were used for the presented autonomous excavation trials. Images of these piles are shown in Fig. 4. The rock pile consisted of a mixture of mud, fine gravel, and large fragments of blasted rock $(30$ to $70 \mathrm{~cm}$ in nominal diameter), which is representative of a real rock pile found in underground mining. The gravel pile consisted of dry gravel with small rocks. In general, gravel piles are "easier" to dig in than rock piles.

\subsection{Autonomous Excavation Trial Procedure}

The admittance controller was only executed during the digging phase of the process (i.e., after the pile had been penetrated). However, for consistency between trials, much of the machine initialization was automated. A Finite State Machine 


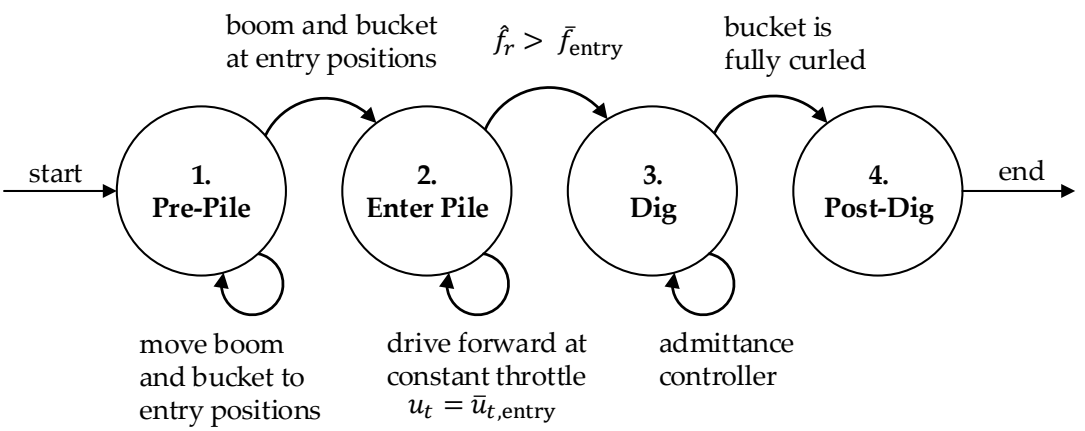

Fig. 5 A Finite State Machine (FSM) representation of the autonomous excavation process. Note that the admittance controller is only executed in the Dig state.

(FSM) representation of this process is shown in Fig. 5. At the start of each trial, the LHD was manually positioned in front of the pile, the boom and bucket were automatically positioned at their entry positions (similar to the configuration shown in Fig. 2), followed by driving forward at a constant throttle $u_{t}=\bar{u}_{t \text {, entry }}$ to penetrate pile and dig using the admittance controller. The automated process ended when the dump cylinder was fully extended. The operator then manually reversed the LHD out of the pile and raised the boom to weigh the excavated payload. Note that both the rock and gravel piles had finite amounts of material, so the material was dumped back onto the pile after weighing.

\section{Field Experiments}

This section presents the results of full-scale autonomous vehicle field experiments conducted to validate the iterative learning-based admittance controller described in Section 2.

\subsection{Setting Control Parameters}

The iterative learning functions (9) and (10) are used to automatically adjust the admittance controller's dump cylinder velocity input parameter $\bar{v}_{d}$ and throttle input parameter $\bar{u}_{t}$. There are also other parameters, listed in Table 1 , that must be set. These parameters were found to be less critical for bucket filling, and do not require re-tuning for different materials. The Enter Pile parameters $\bar{f}_{\text {entry }}$ and $\bar{u}_{t \text {,entry }}$ were determined through preliminary excavation trials. The parameter values were tuned to achieve the earliest possible detection of pile penetration. The control parameters $K_{A}$ and $N$ were tuned until satisfactory digging behaviour was observed, which included desirable response of bucket velocity commands to sudden changes in the measured forces. An example of this is shown in Fig. 6. Note how the target force follows the nominal measured force trajectory. 
Table 1 Gain and parameter choices for field trials

\begin{tabular}{lcc}
\hline Gain/parameter & Description & Value \\
\hline $\bar{f}_{\text {entry }}$ & Dig start force threshold & $2.5 \mathrm{MN}$ \\
$\bar{u}_{t, \text { entry }}$ & Enter Pile throttle & 0.5 \\
$K_{A}$ & Admittance controller gain & 0.03 \\
$N$ & Target Force filter window size & 9 \\
$\alpha$ & Learning rate for $\bar{v}_{d}$ & 0.005 \\
$\beta$ & Learning rate for $\bar{u}_{t}$ & -0.05 \\
{$\left[\bar{v}_{d, \text { min }}, \bar{v}_{d, \max }\right]$} & Learning limits for $\bar{v}_{d}$ & {$[0.01,0.07] \mathrm{m} / \mathrm{s}$} \\
{$\left[\bar{u}_{t, \text { min }}, \bar{u}_{t, \text { max }}\right]$} & Learning limits for $\bar{u}_{t}$ & {$[0,0.5]$} \\
\hline
\end{tabular}
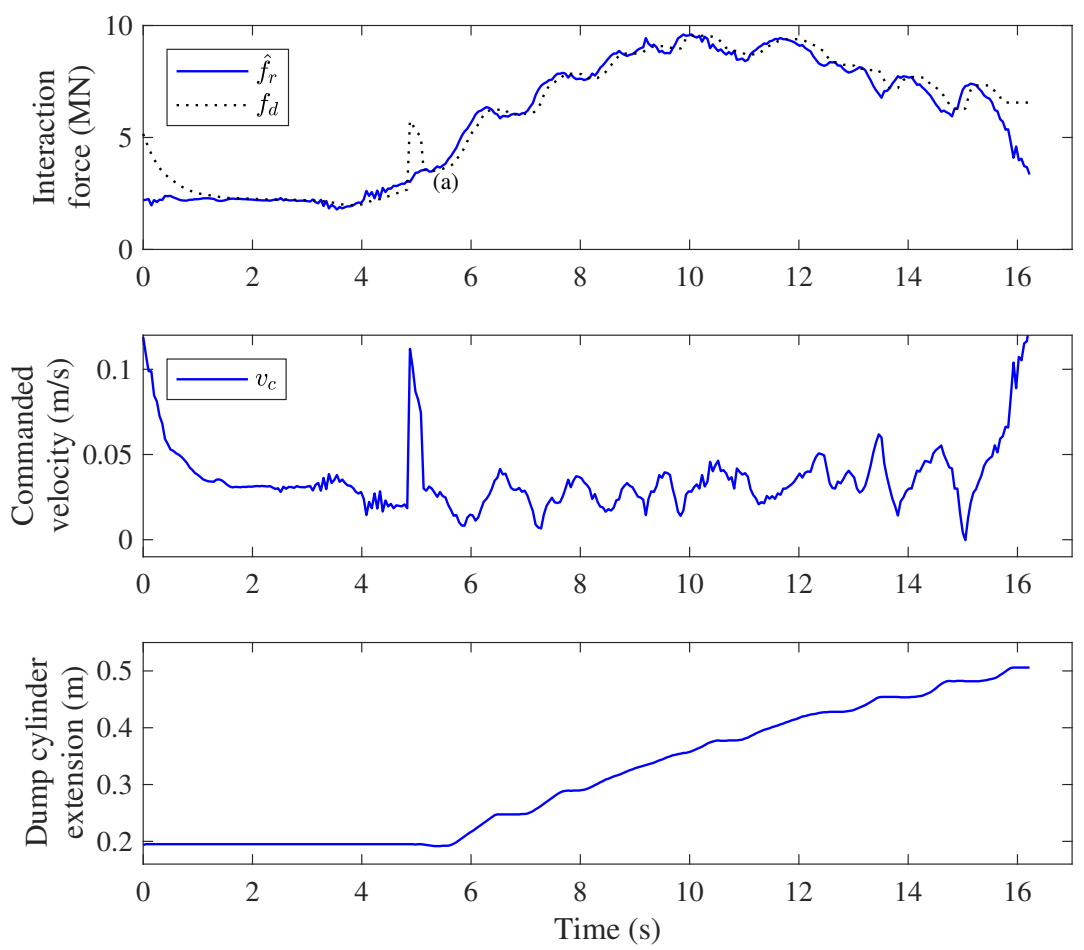

Fig. 6 An example of good digging behaviour as observed through measured force and motion signals. In this case, The admittance controller is executed at approximately the $5.3 \mathrm{~s}$ mark indicated by (a). It was found that $K_{A}=0.02$ and $N=9$ provided the best digging behaviour for the experiment setup.

\subsection{Tuning Iterative Learning Gains}

To estimate stable learning rates $\alpha$ and $\beta$, according to (7), we constructed a linear model of the process based on parameter varying data obtained in our earlier work [8]:

$$
W=\Theta_{0}+\Theta_{1} \bar{v}_{d}+\Theta_{2} \bar{u}_{t},
$$




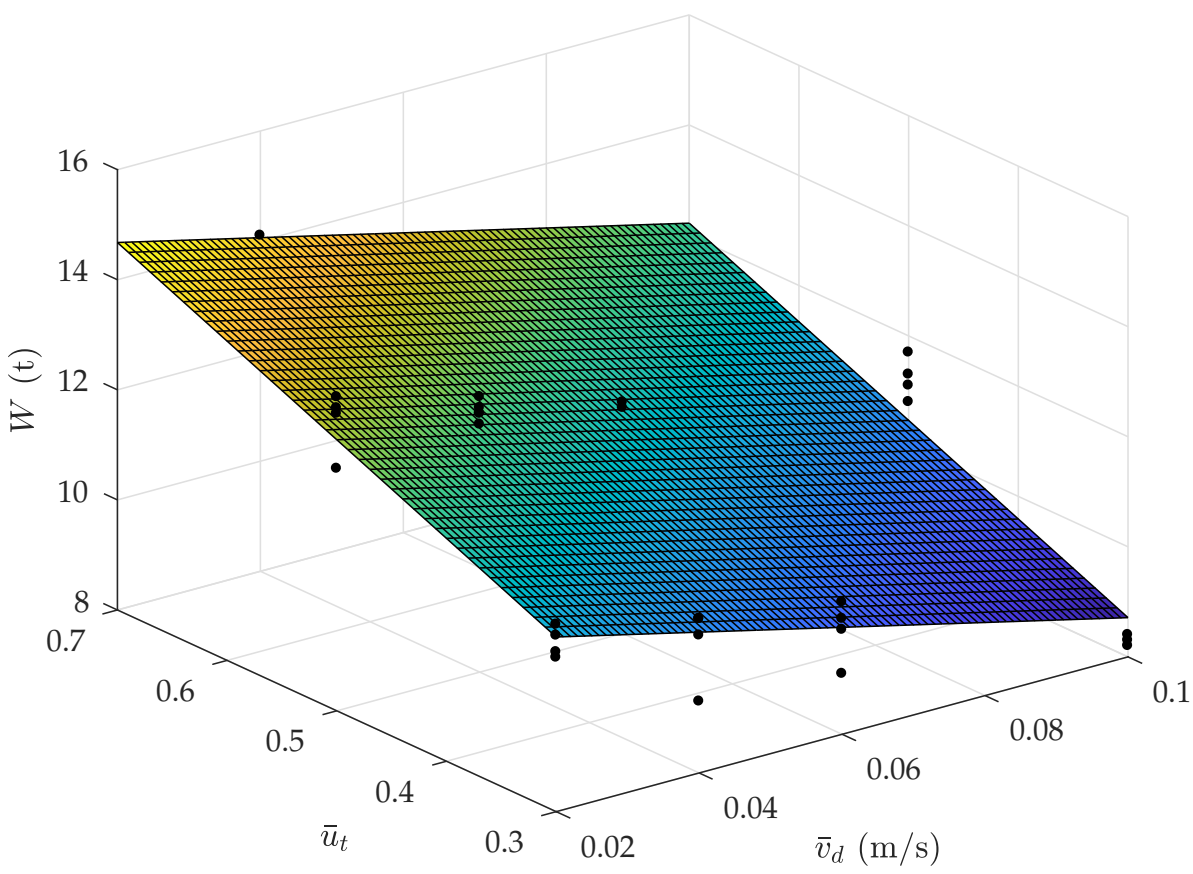

Fig. 7 A linear model of autonomous excavation process developed from regressing data obtained from parameter varying autonomous excavation trials with the rock pile and $7.8 \mathrm{~m}^{3}$ bucket [8].

where $\Theta_{0}, \Theta_{1}, \Theta_{2}$ are model parameters that are obtained through linear regression over the data set. A plot of the linear excavation model is shown in Fig. 7. Note that the data used to construct this model was obtained through autonomous excavation trials with the rock pile and a $7.8 \mathrm{~m}^{3}$ bucket equipped to the $\mathrm{ST} 14$. The root-mean-square-error (RMSE) for the model is 0.6 tonnes, which is good considering the highly variable material characteristics in the rock pile. This model's parameters are $\Theta_{0}=9.16, \Theta_{1}=-30.8$ and $\Theta_{2}=8.75$. Using (7), learning is asymptotically stable if

$$
\left|1+\Theta_{1} \alpha+\Theta_{2} \beta\right|<1 .
$$

By substituting $\Theta_{1}$ and $\Theta_{2}$ from the model and selecting learning rates from Table 1 , the left-hand side of $(12)$ becomes $0.41<1$; i.e., stable. Of course, these parameters are only valid for the acquired data set. Still, this analysis provides a starting point for tuning the learning gains.

From preliminary excavation experiments, we determined the operating ranges for $\bar{v}_{d}$ and $\bar{u}_{t}$. The iterative learning algorithm was saturated to operate within the limits

$$
\begin{aligned}
& \bar{v}_{d, \text { min }} \leq \bar{v}_{d} \leq \bar{v}_{d, \max } \\
& \bar{u}_{t, \min } \leq \bar{u}_{t} \leq \bar{u}_{t, \max } .
\end{aligned}
$$

The dump cylinder reference velocity was limited to values that resulted in reasonable dig times and maximum extension rate. The throttle input parameter $\bar{u}_{t}$ 
was limited to a value that did not result in wheel-slip during digging. The values used for all experiments are provided in Table 1.

\subsection{Iterative Learning Validation Trials}

The iterative learning functions (9) and (10) were validated through full-scale field experiments in two extremely different material characteristics: fragmented rock and gravel. The ST14 machine equipped with a $5.4 \mathrm{~m}^{3}$ bucket and parameter values in Table 1 were used for both material types. The first excavation pass in each material type was performed with manually set initial values for $\bar{v}_{d}, \bar{u}_{t}$. At each successive excavation pass, (9) and (10) were used to automatically update $\bar{v}_{d}, \bar{u}_{t}$ for the specified target weight $W_{d}$. The target weight $W_{d}$ was also varied as the trials progressed to thoroughly test the system.

A total of 34 trials were conducted on the rock pile and 33 trials on the gravel pile. The results for the rock pile trials are presented in Fig. 8, and the results for the gravel pile trials are presented in Fig. 9. In both cases, the iterative learning algorithm was able to update $\bar{v}_{d}$ and $\bar{u}_{t}$ to allow the bucket fill weight to converge to the target fill weight $W_{d}$ in two or three excavation passes. In general, the learning is stable; however, there are larger oscillations in the bucket fill weight for the rock pile due to the high variability of particle sizes in the fragmented rock pile. Also note that for the gravel pile, the algorithm is unable to achieve the target weight $W_{d}=5$ tonnes, even though $\bar{v}_{d}$ is saturated at its maximum value and $\bar{u}_{t}$ is zero. This is due to the amount of material loaded from the initial penetration phase (i.e., the Enter Pile State). Because the gravel pile is relatively easier to dig than the rock pile, approximately six tonnes of material can be loaded from the initial penetration.

\section{Discussion and Conclusions}

In this work we developed and validated, through full-scale field experiments, an iterative learning-based admittance control algorithm for robotic wheel loaders. A simple P-type iterative learning algorithm updates two admittance control parameters - dump cylinder reference velocity parameter $\bar{v}_{d}$ and throttle input parameter $\bar{u}_{t}$-at each excavation pass $j$ based on the error between a target bucket fill weight $W_{d}$ and the measured bucket fill weight $W_{j}$. Field experiments with a 14-tonne capacity load-haul-dump (LHD) machine and two types of excavation material (rock and gravel) revealed that the algorithm is able to achieve a target bucket fill weight within two to three excavation passes. Compared to AI-based approaches that require many training samples [5] and advanced computing hardware, the proposed controller has practical significance as demonstrated through these field experiments.

The stability and convergence rate for the learning algorithm are dependent on tuning two learning gain parameters. In this work, we developed a simple linear model of the autonomous excavation process using previously obtained autonomous excavation data and used this model to select suitable learning gains. However, a priori data is not always available for a particular excavation scenario. Thus, future work should investigate how the learning gains can be adapted based 

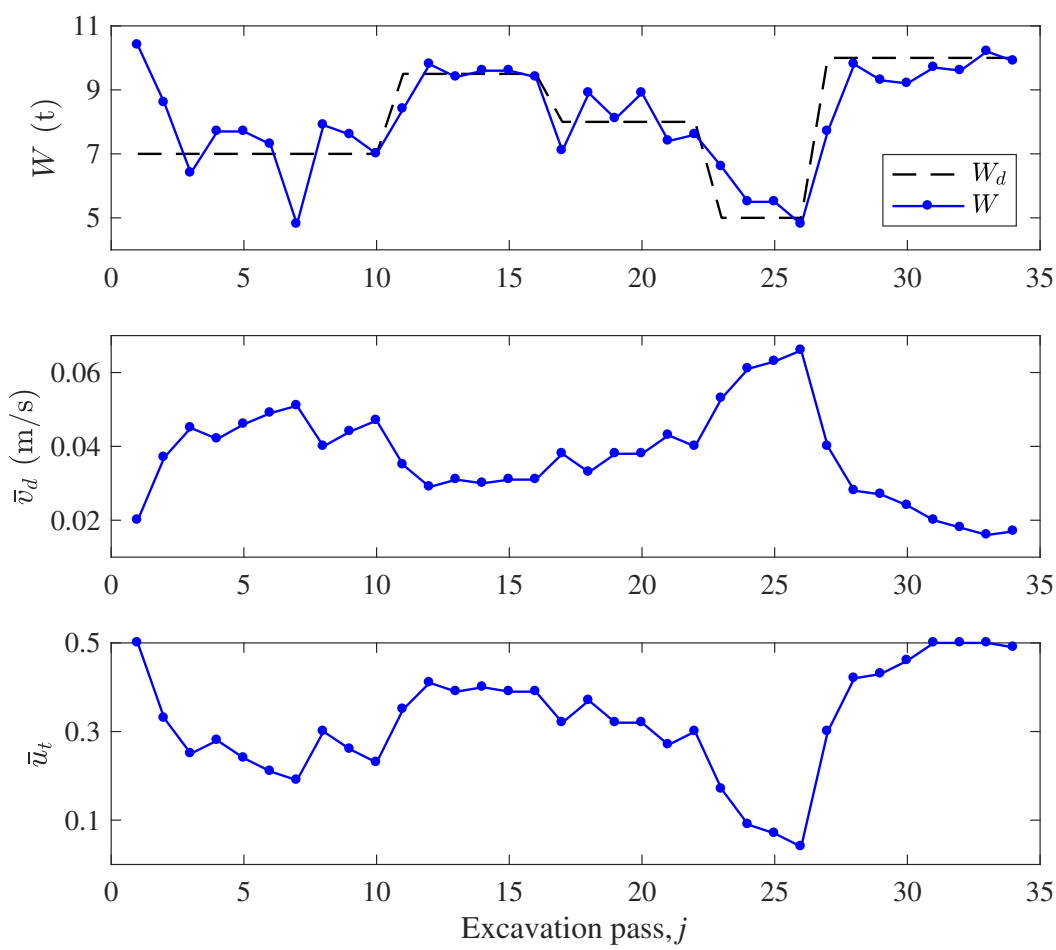

Fig. 8 Results of learning trials with rock pile.

on identification of an improved model of the excavation process. Furthermore, the algorithm should be validated on different wheel loaders and more difficult excavation scenarios (e.g., at operating mines). Although the algorithm was developed and validated using only the Epiroc ST14 machine, we expect that the algorithm is applicable to other underground wheel loaders with similar loading mechanisms.

Acknowledgements The authors would like to thank Håkan Almqvist for his assistance with the field experiments; as well as Ola Petersson, Jörgen Appelgren and Robert Lundh for many useful discussions; and Qing Huang for assistance with programming.

\section{References}

1. Bristow, D.A., Tharayil, M., Alleyne, A.G.: A survey of iterative learning control. IEEE Control Systems Magazine 26(3), 96-114 (2006)

2. Buchli, J., Stulip, F., Theodorou, E., Schaal, S.: Learning variable impedance control. The International Journal of Robotics Research 30(7), 820-833 (2011)

3. Dadhich, S., Bodin, U., Andersson, U.: Key challenges in automation of earth-moving machines. Automation in Construction 68, 212-222 (2016)

4. Dadhich, S., Bodin, U., Sandin, F., Andersson, U.: Machine learning approach to automatic bucket fill. In: Proceedings of 24th Mediterranean Conference on Control and Automation, pp. 1260-1265. Athens, Greece (2016) 

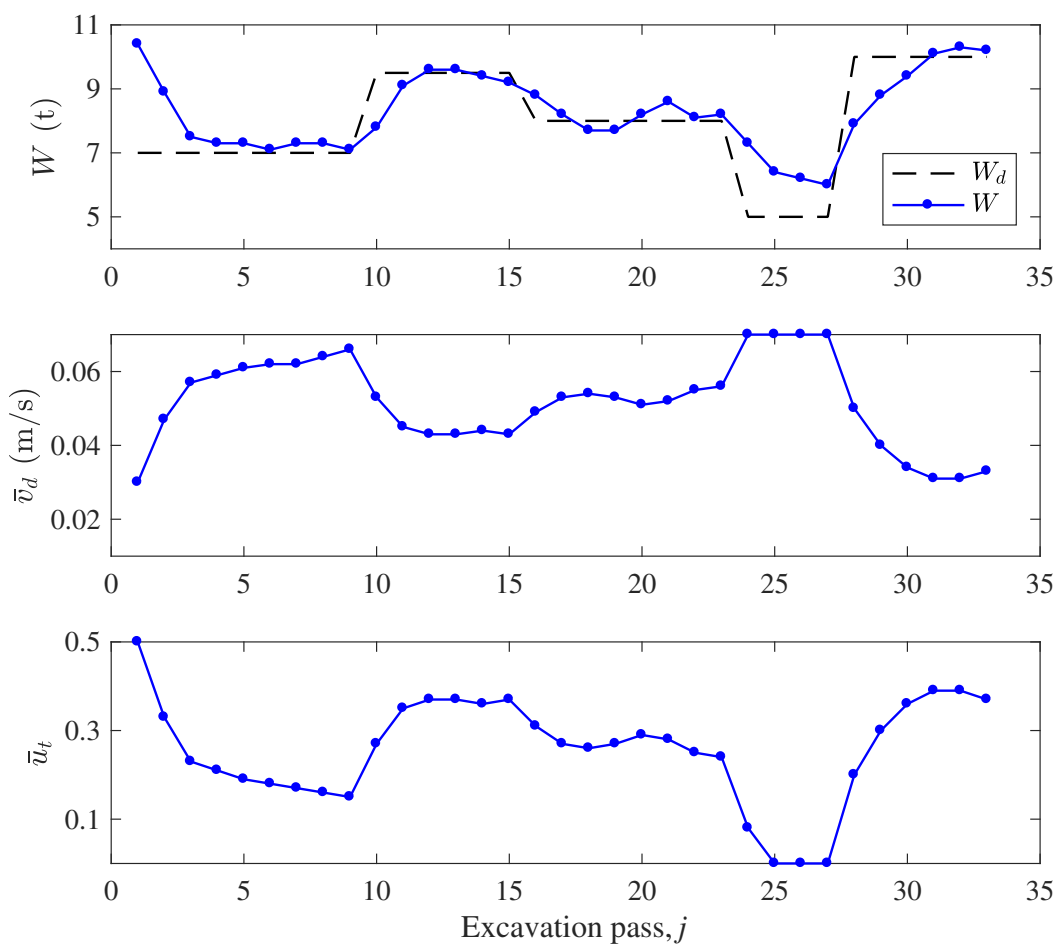

Fig. 9 Results of learning trials with gravel pile.

5. Dadhich, S., Sandin, F., Bodin, U., Andersson, U., Martinsson, T.: Field test of neuralnetwork based automatic bucket-filling algorithm for wheel-loaders. Automation in Construction 97, 1 - 12 (2019). URL https ://doi.org/10.1016/j.autcon.2018.10.013

6. Dimeas, F., Aspragathos, N.: Reinforcement learning of variable admittance control for human-robot co-manipulation. In: Proceedings of the 2015 IEEE/RSJ International Conference on Intelligent Robots and Systems, pp. 1011-1016. Hamburg, Germany (2015)

7. Dobson, A.A., Marshall, J.A., Larsson, J.: Admittance control for robotic loading: Design and experiments with a 1-tonne loader and a 14-tonne LHD. Invited paper in the special issue on Field and Service Robotics in the Journal of Field Robotics 34(1), 123-150 (2017)

8. Fernando, H., Marshall, J.A., Almqvist, H., Larsson, J.: Towards controlling bucket fill factor in robotic excavation by learning admittance control setpoints. In: M. Hutter, R. Siegwart (eds.) Field and Service Robotics: Results of the 11th International Conference, pp. 35-48. Zürich, Switzerland (2017)

9. Goska, W.J., Muller, J.Y., Gaibler, D.W., Thompson, D., Lawe, D.A., Van Schoiack, M.M.: Computerized monitoring management system for load carrying vehicle, US Patent 5659 470 (1997)

10. Haddadin, S., Croft, E.: Springer Handbook of Robotics, 2 edn., chap. 69-Physical humanrobot interaction, pp. 1835-1874. Springer International Publishing, Switzerland (2016)

11. Hemami, A., Hassani, F.: An overview of autonomous loading of bulk material. In: Proceedings of the 26th International Symposium on Automation Robotics in Construction, pp. 405-411. Austin, Texas (2009)

12. Jiménez, M.F., Monllor, M., Frizera, A., Bastos, T., Roberti, F., Carelli, R.: Admittance controller with spatial modulation for assisted locomotion using a smart walker. Journal of Intelligent \& Robotic Systems (2018). URL https://doi.org/10.1007/ s10846-018-0854-0 
13. Kim, Y.J., Seo, J., Kim, H., Kim, K.G.: Impedance and admittance control for respiratorymotion compensation during robotic needle insertion - a preliminary test. The International Journal of Medical Robotic and Computer Assisted Surgery pp. 1-10 (2016)

14. Li, Y., Ge, S.: Impedance learning for robots interacting with unknown envirnonments. IEEE Transactions on Control Systems Technology 22(4), 1422-1432 (2014)

15. Maeda, G.J., Manchester, I.R., Rye, D.C.: Combined ILC and disturbance observer for the rejection of near-repetitive disturbances, with application to excavation. IEEE Transactions on Control Systems Technology 23(5), 1754-1769 (2015)

16. Marshall, J.A., Murphy, P.F., Daneshmend, L.K.: Toward autonomous excavation of fragmented rock: Full-scale experiments. IEEE Transactions on Automation Science and Engineering 5(3), 562-566 (2008)

17. Seraji, H.: Adaptive admittance control: an approach to explicit force control in compliant motion. In: Proceedings of the 1994 IEEE International Conference on Robotics and Automation, pp. 2705-2712. San Diego, California (1994)

18. Villani, L., De Schutter, L.: Springer Handbook of Robotics, 2 edn., chap. 9-Force Control, pp. 195-220. Springer International Publishing, Switzerland (2016)

19. Wang, C., Li, Y., Ge, S.S., Lee, T.H.: Reference adaptation for robots in physical interactions with unknown environments. IEEE Transactions on Cybernetics 47(11), 3504-3515 (2017)

20. Yamawaki, T., Ishikawa, H., Yashima, M.: Iterative learning of variable impedance control for human-robot cooperation. In: Proceedings of the 2016 IEEE/RSJ International Conference on Intelligent Robots and Systems, pp. 839-844. Daejeon, Korea (2016) 